\title{
An Early Warning Monitoring System for Illicit Drug Use at Large Public Events: 'Close to Real Time' Trace Residue Analysis of Discarded Drug Packaging Samples
}

Henry West ${ }^{1}$, John Fitzgerald ${ }^{2}$, Katherine Hopkins ${ }^{1,2}$, Eric Li ${ }^{3}$, Nicolas Clark ${ }^{4,5}$, Stephanie Tzanetis $^{6,7}$, Shaun L. Greene ${ }^{8,9}$, Gavin E. Reid ${ }^{1,10,11 *}$

1. School of Chemistry, The University of Melbourne, Victoria, Australia

2. School of Social and Political Sciences, University of Melbourne, Victoria, Australia

3. Agilent Technologies Australia, Victoria, Australia

4. North Richmond Community Health, Victoria, Australia.

5. Royal Melbourne Hospital, Victoria, Australia.

6. Harm Reduction Victoria, Victoria, Australia

7. Harm Reduction Australia, New South Wales, Australia

8. Victorian Poisons Information Centre, Austin Health, Victoria, Australia

9. Department of Medicine, Faculty of Medicine, University of Melbourne, Victoria, Australia

10. Department of Biochemistry and Pharmacology, The University of Melbourne, Victoria, Australia

11. Bio21 Molecular Science and Biotechnology Institute, The University of Melbourne, Victoria, Australia

*Corresponding author: gavin.reid@,unimelb.edu.au 


\begin{abstract}
Inspired by Locard's exchange principle which states "every contact leaves a trace", we report the development and application of a trace residue sampling and analysis strategy for discarded 'Drug Packaging Samples' (DPS), as part of an early warning monitoring system for illicit drug use at large public events. Using Direct Analysis in Real Time (DART) - mass spectrometry (MS) and -tandem mass spectrometry (MS/MS), rapid and high-throughput identification and characterisation of a wide range of illicit drugs and adulterant substances was achieved, including those present in complex poly-drug mixtures and at low relative abundances, and with analysis times of less than one minute per sample. 1362 DPS were analysed either 'off-site' using laboratory-based instrumentation or 'on-site' in 'close-to-realtime' using a transportable mass spectrometer housed within a mobile analytical laboratory. $92.2 \%$ of DPS yielded positive results for at least one of 15 different drugs and/or adulterants, including cocaine, MDMA, and ketamine, as well as numerous 'novel psychoactive substances' (NPS). Notably, polydrug mixtures were more common than single drugs, with $52.6 \%$ of positive DPS found to contain more than one substance, and with 42 different drug and polydrug combinations observed throughout the study. For analyses performed 'on-site', reports to key stakeholders including event organisers, first aid and medical personnel, and peer-based harm reduction workers could be provided in as little as 5 minutes after sample collection. Then following risk assessment of the potential harms associated with their use, drug advisories or alerts were then disseminated to event staff and patrons, and subsequently to the general public, when substances with particularly toxic properties were identified.
\end{abstract}




\section{Introduction}

The use of psychoactive drugs is common in society, including at large public events such as music and dance festivals. However, due to their unknown origins, compositions and lack of regulation, the use of these substances presents risks of harm, including toxicity, overdose and death $[1,2]$. These risks may be exacerbated when a drug sample has an unknown quantity, or is adulterated via the addition of other pharmaceutically active compounds to dilute, 'bulk up' or otherwise improve the appearance and/or apparent quality of the product [3] e.g., adulteration of cocaine with levamisole and/or lidocaine [4,5]. Additional risk exacerbation occurs when a drug is mis-sold, e.g., counterfeited pharmaceuticals [6,7], or when 'conventional' illicit drugs such as cocaine, MDMA or ketamine, are adulterated with or substituted for structurally related analogues or Novel Psychoactive Substances (NPS) such as synthetic cathinones, synthetic cannabinoids, or novel synthetic opioids such as fentanyl and its analogues [8]. Due to their ever increasing number and structural diversity (up to December 2020, 1,047 substances have been reported to the United Nations Office on Drugs and Crime (UNODC) Early Warning Advisory (EWA) on NPS [9]), the pharmacological properties of many NPS, and their subsequent potential for adverse side-effects are poorly understood [1016]. Therefore, to deliver effective public health and harm reduction interventions at large public events there is a need to rapidly identify and characterise the drugs and drug combinations in use, including NPS that have not previously been reported or characterised $[17,18]$, such that information regarding their presence and/or abundances, and assessment of their potential adverse effects, can be relayed to relevant stakeholders e.g., first aid and medical personnel, peer-based harm reduction workers, and/or event patrons.

Throughout Europe since the 1990's [18,19], more recently in the UK [20,21] and New Zealand [22], and at a single location on two occasions to date in Australia [23,24], drug checking (also known as pill testing) services have been established 'on-site' at music festivals or 'off-site' at fixed site locations within the community, for people who use drugs to be informed about the identity, and in some instances, purity, of drugs in their possession, prior to consumption. In addition to simple colorometric reagent tests, that generally lack sensitivity and specificity to reliably detect or confidently identify the enormous number of substances

currently available, a range of more sophisticated analytical techniques have been employed for drug checking in these services, including Fourier Transform - InfraRed (FT-IR) spectroscopy [25-29]. The potential benefits of FT-IR include minimal sample preparation, minimal sample amount (low mg quantities), speed of analysis ( $<1$ minute per sample), relative 
low establishment cost, and simple operational complexity (i.e., low technical expertise requirement). The presence of the predominant drug and/or other 'inert filler' compounds that are present can be presumptively but confidently determined by searching the resultant data against pre-defined reference libraries of authentic compounds [26]. However, FT-IR is largely a qualitative analysis technique, such that critical quantitative information regarding drug 'purity' or 'dose' is typically not obtained. Furthermore, FT-IR is limited in the detection of potentially highly harmful drugs present at low levels relative to 'inert filler' compounds or other major drugs that may be present (i.e., in poly drug mixtures), or when a drug is not included in the reference library (e.g., new psychoactive substances (NPS)). Raman spectrometers, also used previously for the analysis of illicit substances in field-based drug analysis applications, can suffer from similar limitations [25,30]. Therefore, additional approaches and resources are typically required to provide further and more precise information regarding drug compositions and purity e.g., by using a team of highly-trained chemists to extract and perform detailed chemical analysis of the drugs, to reduce the potential for false negative (and false positive) results.

In contrast, mass spectrometry (MS) is widely acknowledged as the 'gold standard' analytical measurement technology for forensic drug analysis, including for drug monitoring [31,32]. MS, when coupled with established chromatographic separation techniques, namely Gas Chromatography (GC)-MS [33] and Liquid Chromatography (LC)- Electrospray Ionization (ESI)-MS or -Tandem Mass Spectrometry (MS/MS) [34-36] are capable of providing definitive drug identifications with high sensitivity, specificity and quantitative accuracy, including for low level components within poly-drug mixtures, by matching the observed retention times, mass-to-charge ratio's and/or characteristic fragmentation patterns for each drug against the information contained within reference libraries generated from authentic standards [35,36]. MS techniques are also capable of de-novo identifying and characterising novel drug substances that appear on the market. Commercially available 'portable' or 'transportable' GC-MS instrumentation have also been employed for on-site drug testing applications, e.g., at clandestine laboratories by police forensic services, or off-site in drug checking services. However, these 'hyphenated' MS methods can suffer from relatively low throughput capability due to the need for time consuming sample extraction/processing steps and long chromatographic analysis times (often 10's of minutes per sample), can have relatively high establishing or ongoing operating costs, and require significant expert technical knowledge for operation and maintenance. Therefore, they are not considered fit for purpose for use in drug checking applications requiring high throughout, or in 'close to real time' harm 
reduction initiatives deployed in varied settings such as music festivals or supervised drug consumption facilities.

As a means to address these limitations, various alternative MS based strategies employing 'ambient ionisation' techniques for direct sample introduction have been investigated [32], including Desorption ElectroSpray Ionization (DESI) [37,38], Direct Analysis in Real Time (DART) [39-42], Low Temperature Plasma (LTP) ionization [43-45], Paper-Spray (PS) ionization [46-49], and Atmospheric Solids Analysis Probe (ASAP) [50]. Importantly, these approaches enable rapid MS and MS/MS data acquisition with little or no requirement for sample preparation or separation prior to sample introduction [32], with sufficient sensitivity for trace-level analysis, and can be interfaced with portable mass spectrometry instrumentation $[37,38,48,50,51]$ for direct qualitative or quantitative analysis of pharmaceutical and illicit drug substances including synthetic cathinones [37] and fentanyl and fentanyl-analogues [49], including when in the presence of other illicit drugs at significantly higher concentration [42].

Here, as part of developing an early warning monitoring system for illicit drug use, including for 'close to real time' applications at large public events, we describe the development and application of an approach employing trace-residue sampling of discarded 'Drug Packaging Samples' (DPS) followed by high-throughput and rapid qualitative identification and characterisation of illicit drug substances found within, using DART-MS and -MS/MS on both laboratory-based ultra-high-resolution and accurate mass spectrometry (UHRAMS) instrumentation, as well as in a field deployed mobile analytical laboratory using a transportable triple quadrupole mass spectrometer instrument. Importantly, the results from

this study are shown to enable rapid risk assessments of the potential harms associated with the identified substances, with subsequent dissemination of drug alerts or advisories to event staff and patrons, and to the general public, when substances with particularly toxic properties were identified.

\section{Methods}

\section{Sample collection}

1362 samples tentatively assigned as discarded 'Drug Packaging Samples' (DPS) were collected during routine waste collection at multiple large public events including music / dance festivals and other entertainment events ranging in size from 2,000 - 80,000 people, as 
well as other public locations where illicit drug use was likely to occur, throughout metropolitan Melbourne and regional Victoria, Australia between September 2019 and March 2020, and in November, 2020. The estimated combined attendance at these events was $>200,000$ people. Approval for the project was granted by the University of Melbourne Human Research Ethics Committee, and authority for the collection and storage of the illicit drug samples of interest was granted via a permit to University of Melbourne Bio21 Molecular Science and Biotechnology Institute, under the Drugs, Poisons and Controlled Substances Act 1981. The types of DPS obtained are listed in Table 1, with reusable, re-sealable plastic 'ziplock' bags being the most prevalent (90\%), consisting of a variety of sizes and with a variety of distinguishing manufacturers or distributors features such as line thickness and colour, and branding or 'logos'. Notably, $86 \%$ of DPS contained no visible residue, $11 \%$ were classified as containing visible residue (defined as containing trace amounts of powder or crystals) and only 3\% had 'measurable amounts' (defined as containing greater than $5 \mathrm{mg}$ of material). Due to the environments from which they were collected, DPS were potentially exposed to a range of conditions and contaminants, including heat, water, dirt, sand, urine, food waste, etc.

Table1. Summary of drug packaging sample (DSP) types.

\begin{tabular}{|l|l|l|l|l|}
\hline Sample type & No visible residue & Visible residue & 'Measurable' & Total \\
\hline Ziplock bag & 1061 & 134 & 32 & 1227 \\
\hline Clingwrap & 37 & 5 & 0 & 42 \\
\hline Foil & 18 & 4 & 0 & 22 \\
\hline $\begin{array}{l}\text { Pill casing/ gel } \\
\text { capsule }\end{array}$ & 18 & 7 & 8 & 33 \\
\hline Other & 35 & 3 & 0 & 38 \\
\hline Total & 1169 & 153 & 40 & 1362 \\
\hline
\end{tabular}

\section{Sample Preparation}

Samples were prepared for analysis by dry swabbing the surface area of the DPS (e.g., the interior of the 'ziplock' bags) using commercially available cotton tip applicators (Swisspers, Kingsgrove, NSW, Australia). For samples containing 'visible residue' and 
'measurable' amounts of material, the cotton tip applicators were manually gently 'flicked' to displace any loose material. The swabs were then cut to a length such that the widest circumference of the head aligned vertically with the tip of the DART probe when placed into the custom sample holder. See the Supplemental Information for additional detail.

\section{Direct Analysis in Real Time (DART) sample introduction}

For all the results described herein, samples were introduced for mass spectrometry analysis using a Direct Analysis in Real Time (DART) source (IonSense, MA, USA) interfaced to the mass spectrometers using a Vapur interface. During initial method development, a number of variables that could affect the practical application and performance of the DART source for trace residue drug analysis were evaluated and optimized (see the Supplemental Information for additional detail). These included the probe tip geometry and size (conicalshaped, $0.5 \mathrm{~mm}$ hole), the probe heater temperature $\left(150^{\circ} \mathrm{C}\right)$, the Vapur interface vacuum ('easy read' needle valve approximately half-way open), the distance between the probe and the entrance of the ceramic transfer tube to the Vapur interface $(3 \mathrm{~cm})$ the distance between the cotton tip and the transfer tube $(3 \mathrm{~cm})$, the distance between the exit of the ceramic transfer

tube and the entrance to the mass spectrometer $(2-3 \mathrm{~mm})$, and the lateral position (one edge of the cotton tip head centred in the path between the probe and transfer tube). Given that the samples were distributed across the entire outside of the cotton tip head, rotation of the cotton tip applicator head containing the samples was manually performed using tweezers to 'spin' the stem of the cotton tip applicator during data acquisition, to ensure even desorption and the acquisition of a mass spectrum that was representative of the entire surface that had been swabbed.

\section{Mass Spectrometry}

Two mass spectrometers were used to obtain the results described herein. For 'off-site' laboratory-based analysis, a Thermo Scientific Q Exactive Plus Orbitrap mass spectrometer (Bremen, Germany) was used. For spectra acquired using the Q Exactive platform, the transfer capillary temperature of the mass spectrometer was set to $250^{\circ} \mathrm{C}$ and the AGC target set to $1 \mathrm{E} 6$. Ions were detected using the Orbitrap mass analyser operating at a mass resolving power of 17,500 (at $200 \mathrm{~m} / \mathrm{z}$ ). MS spectra were acquired in positive ion mode from m/z 100 - 500, and averaged across 100 scans, with a total acquisition time of 7 seconds. HCD-MS/MS spectra 
were collected using an isolation window of between $+/-0.5$ and $1 \mathrm{~m} / \mathrm{z}$, dependent upon signal intensity and isobaric overlap, with the collision energy set between $15-40$. MS/MS spectra were averaged across 100 scans. For 'on-site' close to real time analysis, an Agilent Ultivo triple quadrupole mass spectrometer, housed within a custom modified mobile analytical laboratory, was used. The mobile analytical laboratory was equipped with both mains power and a portable generator to provide power, lighting and air conditioning for climate control, as well as a cold water supply, secure storage cabinets, and heavy-duty laboratory benches for mounting of the mass spectrometry instrumentation and computer (on vibration absorbing rubber pads) and for sample preparation. For spectra acquired using the Agilent Ultivo triple quadrupole mass spectrometer, the mass range was set to $\mathrm{m} / \mathrm{z} 100-500$, with the fragmenter voltage set to $135 \mathrm{~V}$. CID-MS/MS product ion spectra were obtained using unit resolution with a scan dwell time of $500 \mathrm{~ms}$ and an acquisition time of 1 minute.

\section{Identification of illicit drug compounds and adulterants}

Given that the majority of the DPS examined here contained no visible residue, all results in this study are reported as qualitative identifications only (see the Supplemental Information for additional detail regarding the estimated sensitivity of the methodology). For results obtained via 'off-site' laboratory-based analysis, the identities of all drugs and adulterants within DPS were initially assigned based on accurate masses determined from their $[\mathrm{M}+\mathrm{H}]^{+}$ions in positive ionization mode using the Orbitrap (calibrated within 2 ppm mass accuracy), then definitively confirmed by manual comparison of their HCD-MS/MS spectra against reference spectra contained within the mzCloud Advanced Mass Spectral Database [52], or against MS/MS spectra previously reported in the literature. Blank swabs were run every 5 samples as controls. At the MS level of analysis, a positive identification was assigned only if the signal intensity for the precursor ion of interest was 10x the background level in the blank spectra. For 'on-site' close to real time analysis performed using the Agilent Ultivo triple quadrupole embedded within the mobile analytical laboratory, definitive identification of peaks initially observed at nominal $\mathrm{m} / \mathrm{z}$ values corresponding to potential expected drugs, or abundant peaks of unknown species not present in the blanks, was achieved by acquisition of their MS/MS spectra followed by manual comparison against reference spectra contained within the mzCloud Advanced Mass Spectral Database, or against MS/MS spectra previously reported in the literature. 


\section{Results and Discussion}

\section{'Off-site' laboratory-based DART-MS and -MS/MS for rapid trace residue illicit drug monitoring}

Representative spectra acquired by trace residue swabbing and DART-MS analysis of four different DPS ziplock bags, each containing no visible residue, are shown in Figure 1 (the inset to Figure 1A shows a photograph of the clear plastic ziplock bag from which the swab was taken, measuring $30 \times 24 \mathrm{~mm}$, that had been torn open). For the MS spectrum in Figure 1A, tentative assignment of the ions at $\mathrm{m} / \mathrm{z} 194.1174,238.0991$ and 304.1543 were made for 3,4-Methylenedioxymethamphetamine (MDMA) (calculated $\mathrm{m} / \mathrm{z}$ 194.1181), ketamine (calculated m/z 238.0999) and cocaine (calculated m/z 304.1549), respectively, based on close agreement with their molecular formulas, then definitively confirmed by manually matching the product ion $\mathrm{m} / \mathrm{z}$ and relative abundances observed within their HCD-MS/MS product ion spectra against reference spectra of authentic compounds contained in the mzCloud database that had been acquired under approximately the same collision energies (Supplemental Figures S1A-C, respectively). These three drugs were the most commonly observed compounds during our study (see below for further discussion and summary of the overall dataset). MDMA (commonly known as ecstasy or molly) is the drug most commonly associated with recreational use at music festivals and similar events, where it is reported to give rise to user effects including an enhanced sense of well-being and sensory perception $[53,54]$. Ketamine is a dissociative anaesthetic that is commonly consumed for its mild psychedelic effects [55], while cocaine is a stimulant whose use can result in feelings of euphoria [56]. The DPS giving rise to the DART-MS spectra in Figure 1B was found to contain cocaine along with the common adulterants, levamisole and lidocaine (Figure 1B), while those giving rise to the spectra in Figures 1C and 1D were found to contain MDMA adulterated with the substituted cathinones [15], eutylone and ethylone, respectively. Confirmatory HCDMS/MS spectra for each of these adulterants are shown in Supplemental Figure S1D-G (also see below for further discussion regarding these compounds). Importantly, the spectra in Figure 1, obtained by averaging 100 scans, were each acquired in only 7 seconds while the individual HCD-MS/MS spectra were each acquired in only 10 seconds each. Thus, the identity of all the substances in each DPS using this trace residue sampling and analysis approach were definitively confirmed in less than one minute of total analysis time each, indicative of the high-throughput capabilities of DART-MS and MS/MS for illicit drug monitoring. Including 
sample preparation (i.e., swabbing of the DPS) time, it required only two people to analyse up to 80 samples per hour. For these 'off-site' laboratory-based analyses, in which samples were batch collected and then transported to the laboratory for analysis, reporting of results regarding the identity of the drug(s) and/or their packaging appearance were then provided to Event Management Team (EMT) key stakeholders including event organisers, first aid and medical personnel, peer-based harm reduction workers, and security contractors, amongst others, in as little as $60 \mathrm{~min}$ after sample collection, depending upon the distance between the event and the laboratory. Risk assessments of the potential harms associated with the identified substances were then performed by this group, and, as deemed appropriate or necessary, alerts were then provided in 'close to real time' to event personnel, event patrons, and subsequently to the general public (see the section on Substituted Cathinones below), to reduce their risk of drugrelated harm when substances with particularly toxic properties were identified. 

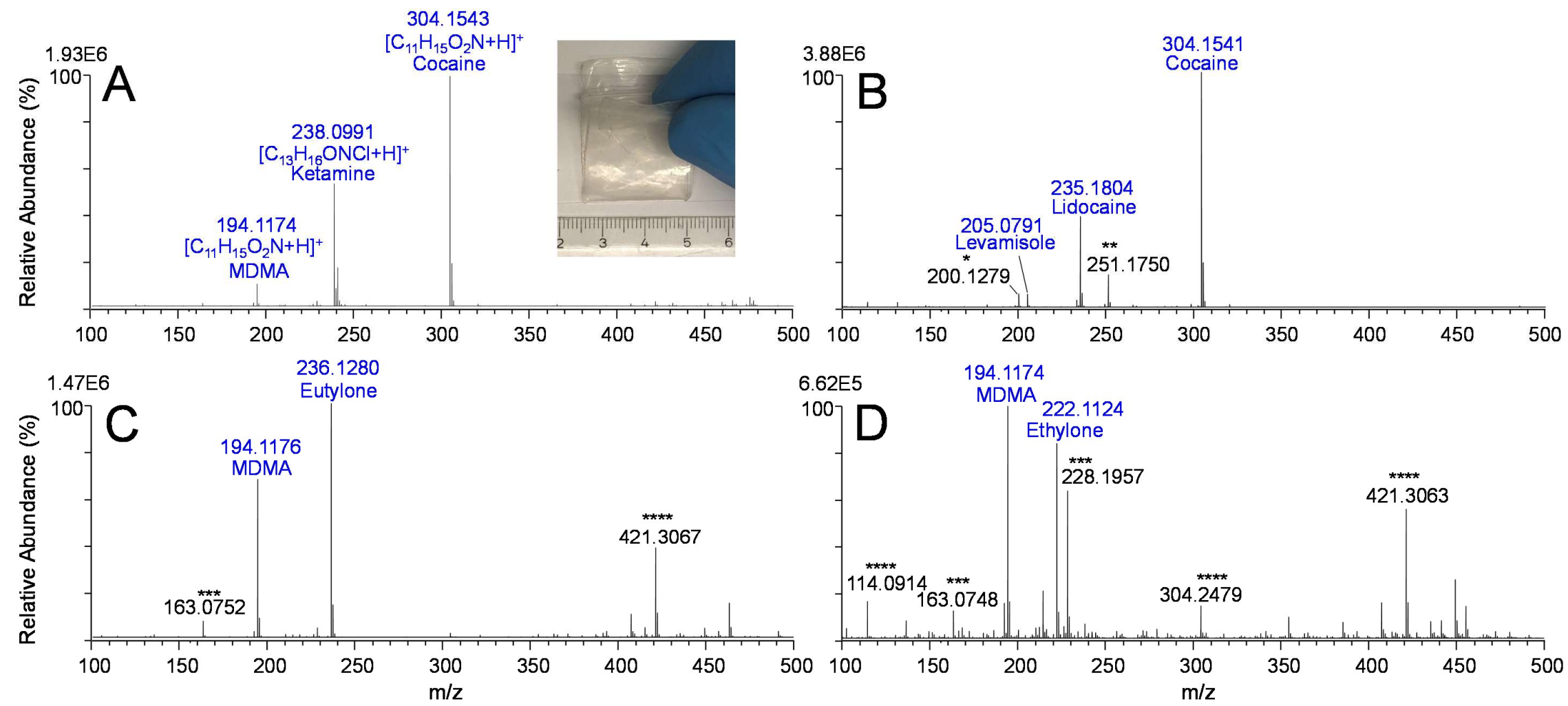

Figure 1. DART-MS spectra from several discarded DPS ziplock bags containing no visible residue, found to contain (A) cocaine, ketamine and MDMA (the inset to panel A shows a photograph of the bag), (B) cocaine adulterated with levamisole and lidocaine, (C) MDMA adulterated with eutylone, and (D) MDMA adulterated with ethylone. * Denotes an in-source cocaine fragment. ** Denotes an insource oxidation product of lidocaine. $* * *$ Denotes an in-source fragment of MDMA **** Denotes background ions. 
An overall summary of the results obtained from the 'off-site' laboratory-based analysis of DPS are shown in Figure 2. A total of 1,315 samples were analysed, with 92.2\% (N=1,212) providing positive results for at least one pharmacologically active compound. A total of 15 different drugs and/or adulterants were identified, including cocaine, MDMA, ketamine, levamisole, lidocaine, methamphetamine, amphetamine, several 'novel psychoactive substances' (NPS) including 2-fluorodeschloroketamine (2-FDCK), tiletamine, the synthetic cathinones ethylone, eutylone and N-ethylpentylone, the endogenous hallucinogen N,Ndimethyltryptamine (DMT), 3,4-methylendioxyamphetamine (MDA), and methylphenidate. Of the DPS samples that tested positive, cocaine (58.9\%) was the most commonly observed drug, followed by MDMA (58.2\%) and ketamine (32.8\%). However, polydrug mixtures were more common than single drugs, with $52.6 \%$ of positive samples containing more than one substance, in 42 different drug combinations. Of the total positive samples, $39.0 \%$ tested for two substances, $12.0 \%$ tested for three active compounds and $1.6 \%$ tested for four active compounds. MDMA/cocaine $\quad(n=227)$, ketamine/cocaine $(n=98)$ and MDMA/ketamine/cocaine $(n=89)$ were the three most common combinations. Although cocaine was observed as the most common drug, it was present only $24.2 \%(n=173)$ of the time as a single drug i.e., without other co-present drugs or adulterants, and was instead found to be co-present with MDMA $48.6 \%$ of the time, and co-present with ketamine $31.2 \%$ of the time. Note that these percentages include all cocaine + MDMA combinations and cocaine + ketamine combinations, including those where cocaine + MDMA + ketamine were all present. Additionally, cocaine was found to be co-present with the common adulterants levamisole (without lidocaine) $9.9 \%$ of the time, co-present with lidocaine (without levamisole) $4.3 \%$ of the time, and co-present with both levamisole and lidocaine $1.3 \%$ of the time. Similarly, MDMA was observed to be present only $40.7 \%$ of the time as a single drug, and $49.2 \%$ of the time to be co-present with cocaine $(n=347)$, and $22.8 \%$ of the time with ketamine. MDMA was also found to be co-present with levamisole (no lidocaine) and with lidocaine (no levamisole) $1.3 \%$ and $1.8 \%$ of the time, respectively, and co-present with both lidocaine and levamisole $0.1 \%$ of the time. In addition, MDMA was found to be co-present $1.3 \%$ of the time with one of the synthetic cathinone drugs, eutylone, ethylone or N-ethylpentylone. Finally, ketamine was observed only $22.4 \%$ of the time as a single drug, while being present $56.0 \%$ of time with cocaine, and $40.5 \%$ of the time with MDMA.

It is particularly notable that we identified in this study a significantly greater fraction of samples that contained polydrug mixtures compared to reports from other recent pill 
testing/drug checking operations in New Zealand and Australian, that could reasonably be expected to share similar drug distribution and/or recreational drug user demographics and usage patterns to those within the events and locations from which the current samples were obtained [22,23]. This difference is likely explained by the different analytical measurement technologies that were employed. For example, in contrast to the study performed here using highly sensitive mass spectrometry-based methods, the results from these other reports were obtained using FT-IR spectroscopy, suggesting a potential bias and/or limitations of that technique against the detection of substances present in polydrug mixtures, particularly those at low levels relative to 'inert filler' compounds or other major drugs that may be present. This highlights the critical requirement for analytical measurement technologies that are capable of accurately identifying and characterising all the components of complex drug mixtures, including those at low relative abundance, if the results are to be used for health promotion and harm reduction purposes. Primarily due to the nature of the sample type (i.e., DPS) and the anonymised collection strategy employed here, it was not feasible to perform direct consultations to confirm whether or not the composition(s) of the identified sample were, or were not, what was expected by the consumer. 


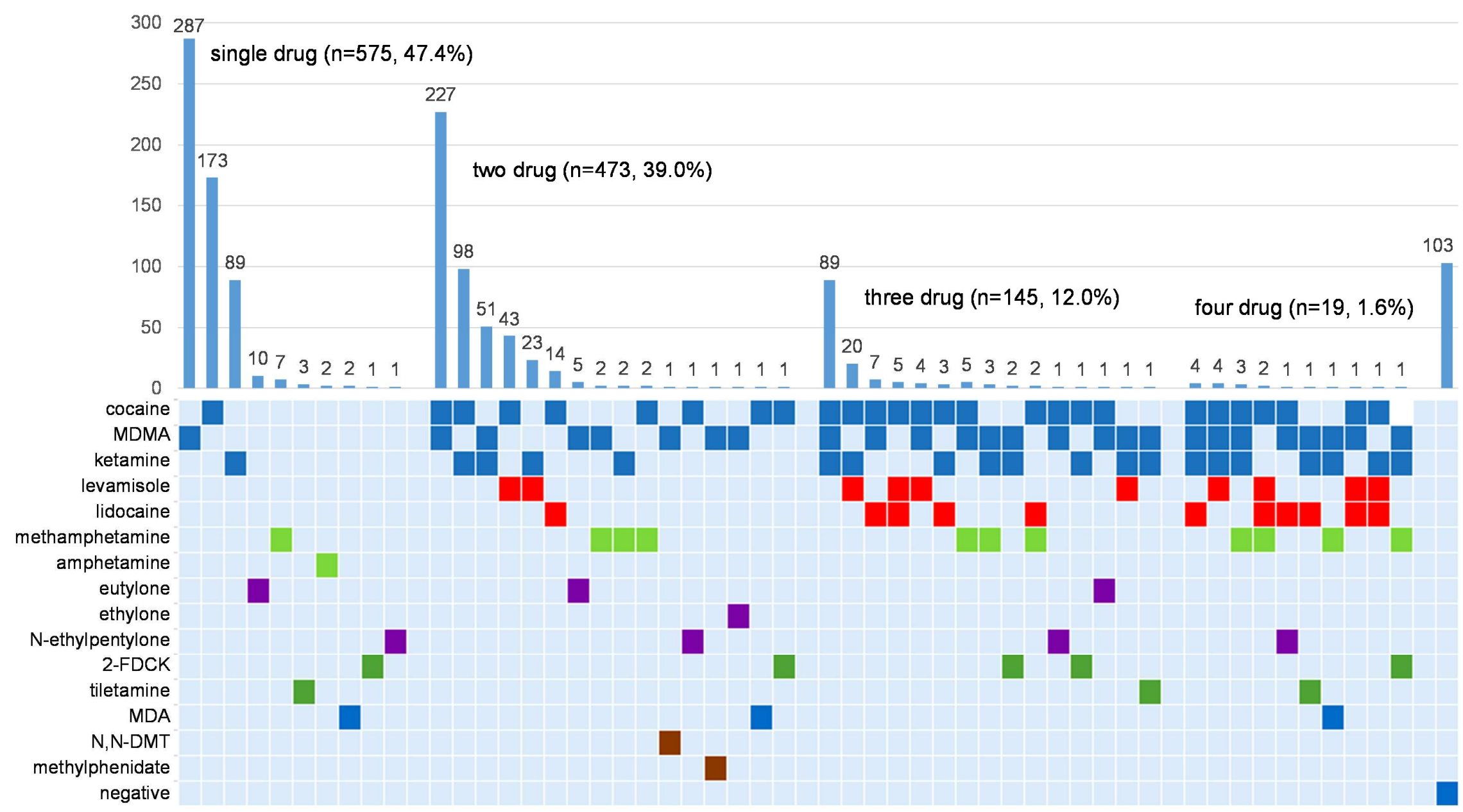

Figure 2. Summary bar graph and matrix of illicit drugs, and drug combinations, observed by trace residue laboratory-based DART-MS monitoring of DPS samples. 


\section{Adulterants and bulking agents}

Lidocaine and levamisole are well-documented adulterants of cocaine [4] and were the two most commonly observed in our study. Lidocaine, a local anaesthetic, is commonly added to cocaine to enhance the characteristic numbing effect that is often associated with 'goodquality' cocaine, while levamisole is used as a bulking and enhancing agent due to its white, fluffy powdery appearance, similar to cocaine. Previously, levamisole was used as a worming agent in humans; however, it was withdrawn from market after being linked to serious cardiovascular issues including agranulocytosis, the depletion of white blood cells, as well as vasculitis leading to inflammation and destruction of blood vessels [57]. Both complications have been reported amongst cocaine users who have consumed cocaine laced with levamisole [5]. Whilst levamisole was primarily observed here to be co-present with cocaine, 23 ketaminecontaining samples also tested positive for the presence of levamisole, revealing the complexity of the illicit market when drugs are readily substituted and the consumers have little awareness of the materials they are consuming. Other adulterants observed in this study included nicotinamide, commonly known as vitamin $\mathrm{B}_{3}$, and dimethylsulfone. Nicotinamide is readily available from most pharmacists and supermarkets, with potential negative effects reportedly occurring only after doses exceeding 3 grams/day [58]. Dimethylsulfone (DMS), a common bulking agent in methamphetamine samples [59], was observed in 5 samples in this component of the study, all of which also contained methamphetamine and corresponding to $17 \%$ of all the methamphetamine samples identified. However, a previous study investigating the impact of DMS on rats did not identify any adverse effects from exposure [60].

\section{Substituted Cathinones}

Substituted cathinones, colloquially known as "bath salts", are synthetic analogues of cathinone, a naturally occurring stimulant found in the plant khat, and are a class of NPS of concern for recreational drug users [61]. A wide range of substituted cathinones have been reported [11,61]. Like most NPS, the pharmacokinetic and pharmacodynamic properties, as well as the short-term and long-term effects of many substituted cathinone's are poorly understood. Common symptoms associated with their use include agitation, tachycardia, paranoia and seizure/tremors, hyperthermia, and multiorgan system failure [61,62]. In a 2015 study of drug use among nightclub/festival-attending young adults in New York City, analysis 
of donated hair samples revealed that of the samples containing MDMA, 47.9\% contained butylone, and $10.4 \%$ contained methylone. Furthermore, the hair samples from $41.2 \%$ of respondents who reported no lifetime use of NPS or unknown pills or powders, tested positive for the synthetic cathinones butylone, methylone, alpha-pyrrolidinovalerophenone ( $\alpha$-PVP), 5or 6-(2-aminopropyl)benzofuran (5/6-APB), or 4-fluoroamphetamine (4-FA) [63]. This suggests that many MDMA-users may be unintentionally using synthetic cathinones or other NPS, and that the risks associated with the use of MDMA may be further exacerbated through adulteration or substitution with synthetic cathinone drugs.

During this study, three substituted cathinones were identified; ethylone, eutylone and $\mathrm{N}$-ethylpentylone. Eutylone was the most commonly observed and was found to be present in 16 samples (10 times as a single drug, 5 times in combination with MDMA (Figure 1C), and once in combination with MDMA and cocaine). Ethylone was observed in only one sample, co-present with MDMA (Figure 1D), while N-ethylpentylone was observed in a total of 4 samples. As a demonstration of the general utility of the cotton tip 'swabbing' method described here for the detection and identification of synthetic cathinone drugs from a variety of DPS materials, including those containing polydrug mixtures and at varying relative abundances, the DART-MS spectra from the four N-ethylpentylone-containing samples are shown in Figure 3. Figure 3A shows the spectrum obtained through swabbing of a ziplock bag, that was found to contain $\mathrm{N}$-ethylpentlyone at moderate relative abundance and co-present with cocaine, MDMA and lidocaine, while Figure 3B shows the result from swabbing a piece of aluminium foil found to contain N-ethylpentylone at low relative abundance and co-present with MDMA and cocaine. In contrast, Figure 3C shows the spectrum obtained by analysis of an intact green, speckled pill imprinted with 'UPS', that was collected as a discarded 'ground find' (also called an "unattached seizure" i.e., a sample not associated with any identifiable individual or police investigation) at a large multi-day music festival. To ensure that the sample subjected to analysis would be representative of the entire pill, the pill was initially manually broken into several small pieces from which representative small scrapings were taken prior to pulverizing into a fine powder, followed by trace residue swabbing. It would be of interest in a subsequent study to systematically examine the heterogeneity associated with the distribution of different drug substance or amounts, in this type of sample. DART-MS and MS/MS (Supplemental Figure S1H) of this sample resulted in the identification of only Nethylpentylone. The risk associated with $\mathrm{N}$-ethylpentylone use is exemplified by a previous case study from a patient who died from an accidental $\mathrm{N}$-ethylpentylone overdose, that reported several complications including hyperthermia, disseminated intravascular coagulation (DIC) 
(blood clots forming throughout cardiovascular system), kidney and liver failure, and with the cause of death being cardiac arrest [2]. In this instance, as the pill had a clearly definable appearance, and following risk assessment of the potential harm associated with this drug at the multi-day event, an initial 'patron alert' was sent via messaging app within 12 hours of its initial identification to warn patrons of its presence, and subsequently a 'public drug alert' was made by the Department of Health \& Human Services State Government of Victoria in March 2020 [64], the first of its kind within the State of Victoria. We note that the specific format and delivery of such alerts is a subject that requires ongoing consideration and evaluation. Finally, the spectra in Figure 3D resulted from analysis of a sample found to contain N-ethylpentylone and cocaine, obtained by swabbing a toilet roll holder in a public toilet cubicle at a popular social location (i.e., not a music/dance festival) on which traces of a white powder was observed. This demonstrated ability to obtain positive identifications from a diversity of DPS materials, as well as directly from surfaces in which drugs had been in contact, indicates that the developed sampling technique is quite versatile, with almost any 'surface' being able to be swabbed. The co-presence of cocaine with three of the four N-ethylpentylone containing samples (and especially in the context of the visible powder on the toilet roll holder from which the sample in Figure 3C was acquired) suggested that the means of administration was likely to be insufflation, which could result in the $\mathrm{N}$-ethylpentylone entering the body at a higher rate and level than from oral ingestion. As a result of this increased potential harm, combined with information regarding a number of $\mathrm{N}$-ethylpentylone associated hospitalisations that occurred around the same period of time as the sample collection, a 'public drug advisory' was subsequently made by the Department of Health \& Human Services State Government of Victoria in December 2020 [65]. 


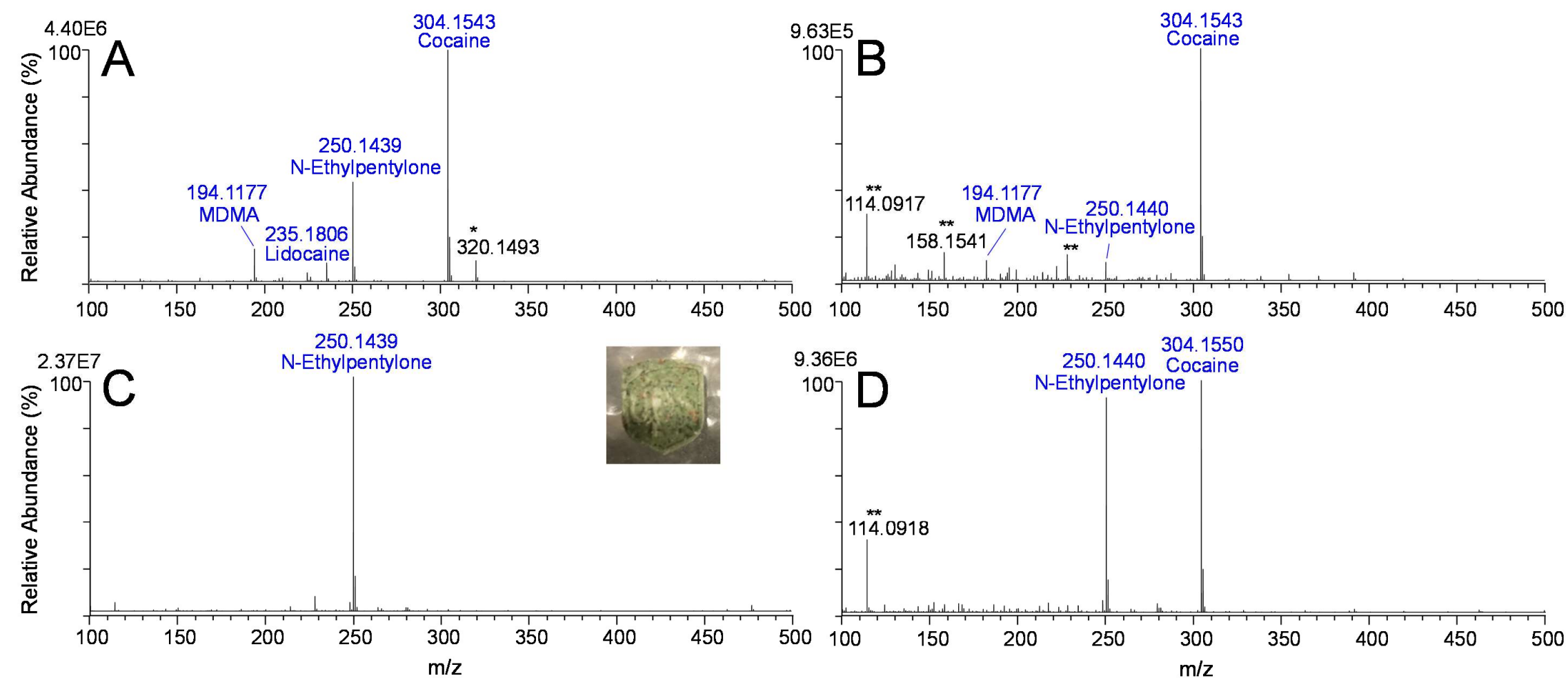

Figure 3. DART-MS of DPS samples containing N-ethylpentylone obtained from dry swabs of (A) a ziplock bag, (B) aluminium foil, (C) a green speckled 'UPS' pill. The inset to panel C shows a photograph of the pill, and (D) residue found on a toilet roll holder in a public toilet cubicle. * Denotes an in-source oxidation product of cocaine. ** Denotes background ions. 


\section{Ketamine analogues}

Two analogues of the dissociative anaesthetic ketamine were identified during this study, namely 2-fluorodeschloroketamine (2-FDCK), and tiletamine. 2-FDCK, an analogue where the chlorine has been substituted with a fluorine [66], was present in six DPS, once by itself, once with cocaine, once with cocaine and ketamine, twice with MDMA and ketamine, and once in a quaternary mixture with methamphetamine, MDMA and ketamine (Supplemental Figure S2A). The co-presence of ketamine in four of the six DPS suggests that the 2-FDCK was introduced as an adulterant or substitution for ketamine. As with many NPS, little is understood regarding the effects, metabolism and potential toxicity of 2-FDCK. However, a study of halogen substitution on metabolism by cytochrome P450 2B6 (the major metabolic enzyme for hepatic metabolism of ketamine, operating through N-demethylation) found that the $\mathrm{k}_{\mathrm{m}}$ was more than double that for 2-FDCK compared to ketamine $(17+/-1$ for ketamine and $40+/-3$ for 2-FDCK) [67], suggesting that 2-FDCK has a lower binding affinity for the enzyme, which may result in a lower metabolism rate. Potential for harm exists therefore, if users re-administer after a period of time, thinking it to be ketamine. Tiletamine, another structural analogue of ketamine in which the chlorophenyl ring is substituted for a thiophene ring, was observed in a total of five samples, three times by itself, once co-present with MDMA and ketamine, and once with MDMA, ketamine and lidocaine (Supplemental Figure S2B). Although tiletamine is often combined with zolazepam (trade name Telazol) for use as a veterinary anaesthesia [68], and human fatalities associated with use of the tiletamine/zolazepam combination have been documented [69], zolazepam was not observed in any of the samples analysed here. The HCD-MS/MS spectra used to definitively confirm the identifications of 2-FDCK and Tiletamine are shown in Supplemental Figures S1I and S1J, respectively.

\section{Identification of other illicit substances}

Methamphetamine, a stimulant and one of the most well-known amphetamines, was observed in 20 DPS in several different combinations, including in quaternary drug mixtures containing i) 2-FDCK, ketamine and MDMA, as shown in Supplemental Figure S2A, and ii) 3,4-Methylenedioxyamphetamine (MDA), MDMA and Ketamine (Supplemental Figure S3A). The confirmatory HCD-MS/MS spectra for methamphetamine and MDA are shown in 
Supplemental Figures S1K and S1L, respectively. MDA, the amphetamine analogue of MDMA, was observed in a total of 4 samples. MDA is the primary metabolite of MDMA, but is also a potential synthetic by-product of MDMA, often observed at very low levels relative to MDMA. In this study however, MDA was observed in three samples without MDMA being present, and was co-present with MDMA at similar intensity in the remaining sample, suggesting its deliberate adulteration or substitution for MDMA. Methylphenidate, commonly known under its trade name 'Ritalin', is a stimulant drug and a first line medication for management of ADHD, that was observed in one DPS co-present with MDMA (see Supplemental Figure S3B, as well as the confirmatory HCD-MS/MS spectrum in Supplemental Figure S1M). With similar reported effects to amphetamines, the recreational use of this substance is well documented [70]. Dimethyltryptamine (DMT), a psychoactive compound related to other psychedelic tryptamines including psilocin and bufotenine, was observed in one sample co-present at low level with MDMA (see Supplemental Figure S3C, and the confirmatory HCD-MS/MS spectrum in Supplemental Figure S1N). Finally, two samples were found to contain amphetamine (an example is shown in Supplemental Figure S3D, with the confirmatory HCD-MS/MS shown in Supplemental Figure S1O).

The potent synthetic hallucinogen $25 \mathrm{C}-\mathrm{NBOMe}$, a member of the phenethylamine Nbenzyl methoxy class of compounds, in combination with 4-fluoroamphetamine was recently found to be responsible for several fatal overdoses in Melbourne, Victoria, Australia throughout July 2016 - January 2017 [71], while another NBOMe, 25I-NBOMe, was implicated in at least one other death in Australia [72]. Fortunately, none of these potentially fatal NBOMe drugs, or 4-fluoroamphetamine, were observed in our study. Furthermore. although several health alerts were recently released in New South Wales, Australia with warnings of possible acetylfentanyl and fentanyl-laced cocaine and ketamine samples [73,74], no fentanyl containing samples were observed in our study.

\section{Proof of concept implementation of on-site 'close to real time' DART-MS and -MS/MS analysis for trace residue illicit drug monitoring}

To reduce / eliminate the time delay between DPS collection and analysis, such that results could be reported in 'close to real time', it was desirable to perform the analyses 'onsite', i.e., proximal to where the sample collection occurs. However, to achieve this, portable or transportable mass spectrometry instrumentation is required. Here, to provide a proof-ofconcept demonstration of the practical utility of our trace residue DPS sampling and analysis 
approach for 'on-site' drug monitoring in 'close to real time', a compact triple quadrupole mass spectrometer equipped with a DART ionization source was installed in a customised mobile analytical laboratory and then securely transported for use at a one-day music festival event (approx. 15,000 patrons) (see Supplemental Figure S4). During this proof-of-concept study 47 DPS were analysed, with 44 samples (93.6\%) testing positive for at least one pharmaceutically active compound. Eight different drugs in 10 combinations were identified, with $79.5 \%$ identified as single drugs and $20.5 \%$ as two-drug mixtures (see Figure 4). MDMA was the most commonly observed compound, in a total of $27(61.4 \%)$ of the positive samples, with $21(77.8 \%)$ containing MDMA alone, and the remainder (6) co-present with ketamine. 12 samples were found to contain ketamine (6 in combination with MDMA, 3 as single drugs, 2 in combination with $2 \mathrm{~F}-\mathrm{DCK}$ and 1 in combination with methamphetamine), and 7 samples were found to contain only cocaine. Albeit a proof-of-concept implementation on a limited scale, this is the first report describing the deployment of a 'transportable' DART-MS system interfaced with a compact triple quadrupole mass spectrometer for on-site close to real time drug monitoring at music festivals or large public events, anywhere in the world.

Examples of the DART-MS and -MS/MS spectra resulting from analysis of DPS collected and analysed on-site at this event are shown in Figure 5 and Supplemental Figure S5. The first (Figures 5A and 5B) was from a sample containing no visible residue, that was found to contain cocaine, while a second (Figure 5C and 5D), resulting in the identification of methiopropamine, was acquired from a swab taken from a discarded 'ground find' consisting of a ziplock bag containing four capsules that themselves contained off-white coloured powder. Methiopropamine is a structural analogue of methamphetamine where the phenyl ring has been substituted with a thiophene ring. In this case, the similarity in the experimentally observed CID-MS/MS fragmentation behaviour for methiopropamine (Figure 5D) with other amphetamines, especially methamphetamine, enabled its identity to be initially assigned de novo, despite us not having observed this drug throughout our previous 'off-site' laboratorybased study, then unambiguously confirmed by comparison with the reference spectra for methiopropamine contained within the mzCloud database. In this instance, less than 5 minutes elapsed between running the unknown sample and its identification, and subsequent reporting to on-site medical and first-aid personnel. Similar to other NPS observed in this study, the pharmacology and toxicological effects of methiopropamine, first detected in 2011 in Finland, are largely unknown, but it has been loosely characterised as being amphetamine-like, but with hallucinatory effects. Notably, although acute toxicity related to use of methiopropamine was first reported in the UK in 2014 [75], and a fatality associated with the use of methiopropamine 
was reported in Australia in 2015 [76], this is the first report of the identification of this drug in the context of its potential 'recreational' use at Australian music festivals. A final example of 'on-site' DART-MS and -MS/MS for analysis of a second discarded 'ground find' collected at the event, consisting of a ziplock bag containing a single red coloured pill, resulting in the identification of MDMA, is shown in Supplemental Figure S5. Collectively, these results clearly highlight the potential for 'on-site' transportable mass spectrometry-based instrumentation to definitively determine the identity of new and potentially harmful compounds in close to real time as part of an early warning system for monitoring and responding to illicit drug use at large public events.

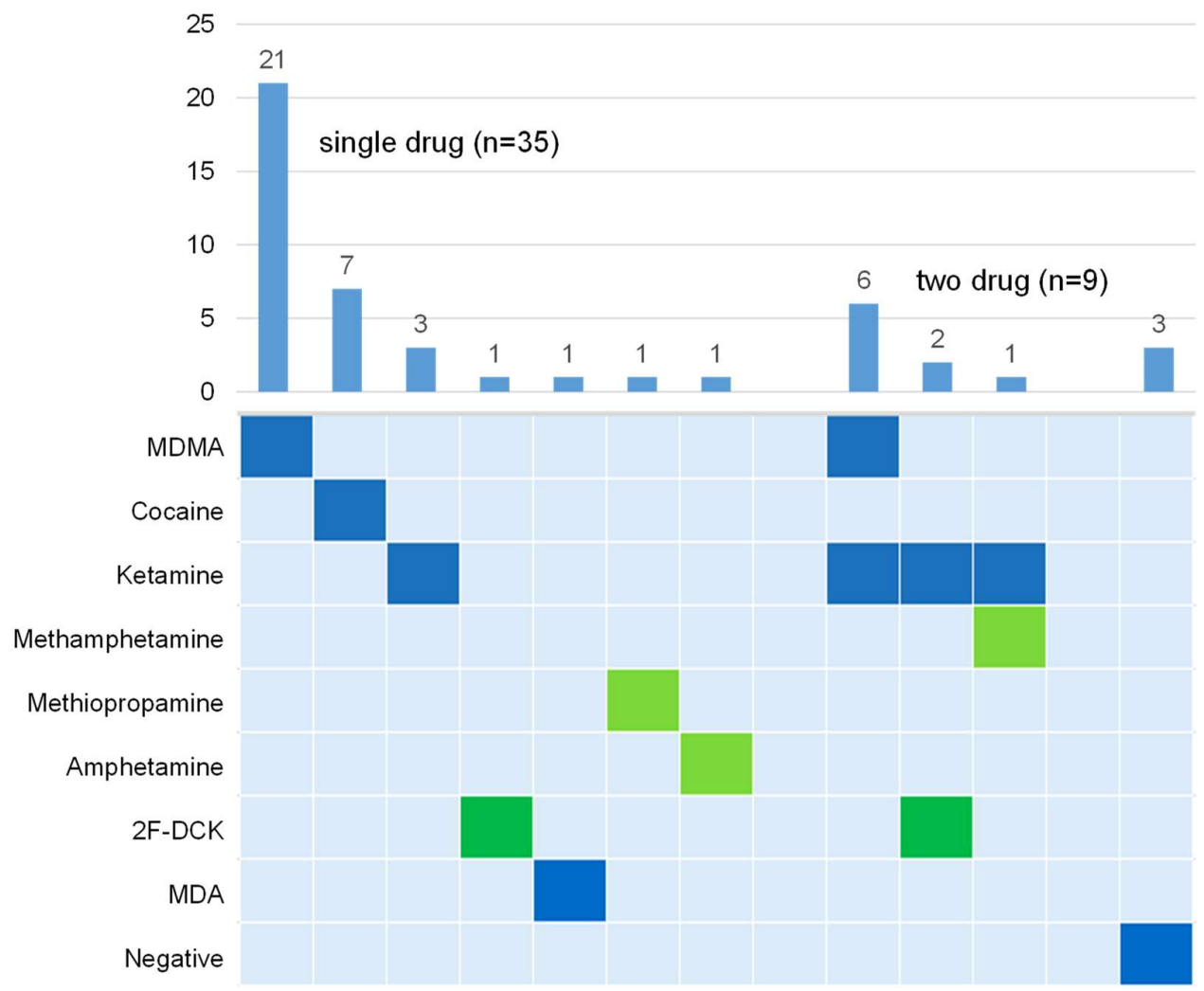

Figure 4. Summary bar graph and matrix of illicit drugs and drug combinations observed from on-site 'close to real time' mobile laboratory-based DART-MS monitoring of DPS samples. 

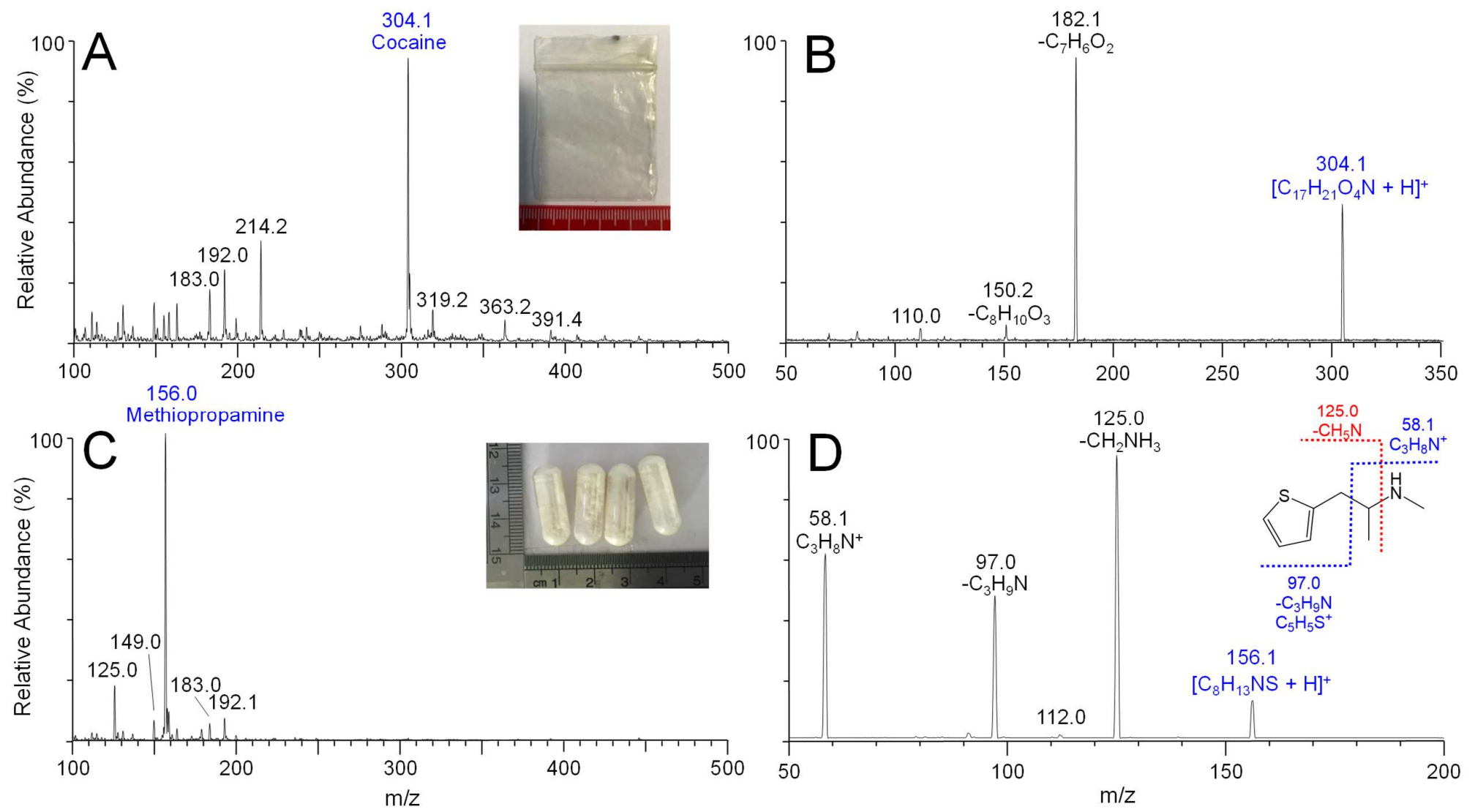

Figure 5. On-site 'close to real time' DART-MS and -MS/MS analysis of illicit drug samples using a transportable triple quadrupole mass spectrometer housed within a mobile analytical laboratory. (A) MS spectrum of a ziplock bag with no visible residue, found to contain cocaine (the inset shows a photograph of the bag). (B) CID-MS/MS spectrum of cocaine (m/z 304.1) from panel A. (C) MS spectrum of a capsule containing an off-white power, found to contain methiopropamine (the inset shows a photograph of the bag). (D) CIDMS/MS spectra of methiopropamine (m/z 156.0) from panel C. 


\section{Conclusions}

The use of illicit drug substances carries risks that can be exacerbated by adulteration or misidentification, or when present in complex poly-drug mixtures. Of particular relevance to the rationale for developing the sample analysis strategy reported herein, including the capability for being deployed 'on-site' and in 'close to real time', are the dual and interacting challenges of being able to identify illicit substances, particularly NPS, when drug users are both combining substances, and trying out new substances, within the milieu of a rapidly changing illicit drug marketplace. Using DART-MS and -MS/MS for sensitive, rapid and highthroughput 'population' level monitoring of a wide range of illicit drug substances found within discarded DPS, we determined that the majority of samples collected during our study at large public events contained polydrug mixtures, including NPS, thereby demonstrating that this method has the capacity to address these dual challenges. Importantly, the results obtained are shown to be a critical input for the provision of harm reduction information to first aid and medical personnel and event patrons, and subsequently to the general public, when substances with particularly toxic properties, or in particular harmful combinations, are identified.

\section{Acknowledgements}

We would like to thank Graham Hilder for his expert assistance with customization and fit-out of the mobile analytical laboratory used in this study. We also gratefully acknowledge Jim Tsiotinas and Tom Hennessy (Agilent Technologies Australia) for collaborating with us and loaning the Ultivo triple quadrupole mass spectrometer that was deployed for use in the mobile analytical laboratory during the on-site 'close to real time' monitoring component of this study. Funding for this study was provided by the University of Melbourne Proof of Concept Fund and from the Victoria State Government Department of Health.

\section{References}

1. Degenhardt L, Hall W. Extent of illicit drug use and dependence, and their contribution to the global burden of disease. Lancet. 2012, 379, 55-70. 
2. Thirakul P, S Hair L, L Bergen K, M Pearson J. Clinical Presentation, Autopsy Results and Toxicology Findings in an Acute N-Ethylpentylone Fatality. J Anal Toxicol. 2017, $41,342-346$.

3. Broséus J, Gentile N, Bonadio Pont F, Garcia Gongora JM, Gasté L, Esseiva P. Qualitative, quantitative and temporal study of cutting agents for cocaine and heroin over 9 years. Forensic Sci Int. 2015, 257, 307-313.

4. Kudlacek O, Hofmaier T, Luf A, Mayer FP, Stockner T, Nagy C, Holy M, Freissmuth M, Schmid R, Sitte HH. Cocaine adulteration. J Chem Neuroanat. 2017, 83-84, 75-81.

5. Zhu NY, Legatt DF, Turner AR. Agranulocytosis after consumption of cocaine adulterated with levamisole. Ann Intern Med. 2009, 150, 287-289

6. Green TC, Gilbert M. Counterfeit Medications and Fentanyl. JAMA Intern Med. 2016, Oct 1;176(10):1555-1557.

7. Arens AM, van Wijk XM, Vo KT, Lynch KL, Wu AH, Smollin CG. Adverse Effects From Counterfeit Alprazolam Tablets. JAMA Intern Med. 2016, 176, 1554-1555.

8. Giné CV, Espinosa IF, Vilamala MV. New psychoactive substances as adulterants of controlled drugs. A worrying phenomenon? Drug Test Anal. 2014, 6, 819-24.

9. https://www.unodc.org/LSS/Home/NPS

10. Hohmann N, Mikus G, Czock D. Effects and risks associated with novel psychoactive substances: mislabeling and sale as bath salts, spice, and research chemicals. Dtsch Arztebl Int. 2014, 111, 139-147.

11. Elliott S, Evans J. A 3-year review of new psychoactive substances in casework. Forensic Sci Int. 2014, 243, 55-60.

12. Armenian P, Vo KT, Barr-Walker J, Lynch KL. Fentanyl, fentanyl analogs and novel synthetic opioids: A comprehensive review. Neuropharmacology. 2018, 134, 121-132.

13. Ifeagwu SC, Raithelhuber M, Crean C, Gerostamoulos D, Chung H, Tettey JN. Toxicology in international drug control-Prioritizing the most harmful, persistent and prevalent substances. Forensic Sci Int. 2017, 274, 2-6.

14. Dargan PL, Wood DM. (Editors). Novel Psychoactive Substances. 2013, Academic Press.

15. Miotto K, Striebel J, Cho AK, Wang C. Clinical and pharmacological aspects of bath salt use: a review of the literature and case reports. Drug Alcohol Depend. 2013, 132, $1-12$. 
16. Helander A, Bäckberg M, Hultén P, Al-Saffar Y, Beck O. Detection of new psychoactive substance use among emergency room patients: results from the Swedish STRIDA project. Forensic Sci Int. 2014, 243, 23-29.

17. Brunt TM, Nagy C, Bücheli A, Martins D, Ugarte M, Beduwe C, Ventura Vilamala M. Drug testing in Europe: monitoring results of the Trans European Drug Information (TEDI) project. Drug Test Anal. 2017, 9, 188-198.

18. Brunt TM, Niesink RJ. The Drug Information and Monitoring System (DIMS) in the Netherlands: implementation, results, and international comparison. Drug Test Anal. 2011, 3, 621-634.

19. Hondebrink L, Nugteren-van Lonkhuyzen JJ, Hunault CC, van den Berg J, van der Gouwe D, van Riel AJHP. New psychoactive substances (NPS) in the Netherlands: occurrence in forensic drug samples, consumer drug samples and poisons center exposures between 2013 and 2017. Addiction. 2020, 115, 716-725.

20. Measham FC. Drug safety testing, disposals and dealing in an English field: Exploring the operational and behavioural outcomes of the UK's first onsite 'drug checking' service. Int J Drug Policy, 2019, 67, 102-107.

21. Measham F. City checking: Piloting the UK's first community-based drug safety testing (drug checking) service in 2 city centres. Br J Clin Pharmacol. 2020, 86, 420-428.

22. Know Your Stuff NZ. 2019 - 2020 Testing report. 2020. Available from: https://knowyourstuff.nz/our-results-2/testing-results/testing-reports/2019-2020testing-report/

23. Vumbaca G, Tzanetis S, McLeod M, Caldicott D. Report on the 2nd Canberra GTM Pill Testing Service. 2019 Harm Reduction Australia. Available from: https://pilltestingaustralia.com.au/wp-content/uploads/2019/08/Report-on-the-2ndACT-GTM-Pill-Testing-Pilot.pdf.

24. Olsen A, Wong G, McDonald D. ACT Pill Testing Trial 2019: Program Evaluation 2019 Australian National University: Canberra ACT. Available from: https://openresearch-repository.anu.edu.au/handle/1885/195646

25. Green TC, Park JN, Gilbert M, McKenzie M, Struth E, Lucas R, Clarke W, Sherman SG. An assessment of the limits of detection, sensitivity and specificity of three devices for public health-based drug checking of fentanyl in street-acquired samples. Int J Drug Policy. 2020, 77, 102661. 
26. Grobério TS, Zacca JJ, Botelho ÉD, Talhavini M, Braga JWB. Discrimination and quantification of cocaine and adulterants in seized drug samples by infrared spectroscopy and PLSR. Forensic Sci Int. 2015, 257, 297-306.

27. Eliaerts J, Dardenne P, Meert N, Van Durme F, Samyn N, Janssens K, De Wael K. Rapid classification and quantification of cocaine in seized powders with ATR-FTIR and chemometrics. Drug Test Anal. 2017, 9, 1480-1489.

28. Bukowski EJ, Monti JA. FTIR-ATR spectroscopy for identification of illicit drugs seized from clandestine laboratories. American Laboratory. 2007, 39, 16-19.

29. Hughes J, Ayoko G, Collett S, Golding G. Rapid quantification of methamphetamine: using attenuated total reflectance fourier transform infrared spectroscopy (ATR-FTIR) and chemometrics. PLoS One. 2013, 8, e69609.

30. West MJ, Went MJ. Detection of drugs of abuse by Raman spectroscopy. Drug Test Anal. 2011, 3, 532-538.

31. Harper L, Powell J, Pijl EM. An overview of forensic drug testing methods and their suitability for harm reduction point-of-care services. Harm Reduct J, 2017, 14, 52.

32. Borden SA, Palaty J, Termopoli V, Famiglini G, Cappiello A, Gill CG, Palma P. Mass Spectrometry Analysis of Drugs of Abuse: Challenges and Emerging Strategies. Mass Spectrom Rev. 2020, 39, 703-744.

33. Armbruster DA, Tillman MD, Hubbs LM. Limit of detection (LQD)/limit of quantitation (LOQ): comparison of the empirical and the statistical methods exemplified with GC-MS assays of abused drugs. Clin Chem. 1994, 40, 1233-1238.

34. Dams R, Murphy CM, Lambert WE, Huestis MA. Urine drug testing for opioids, cocaine, and metabolites by direct injection liquid chromatography/tandem mass spectrometry. Rapid Commun Mass Spectrom. 2003, 17, 1665-1670.

35. Cao Z, Kaleta E, Wang P. Simultaneous Quantitation of 78 drugs and metabolites in urine with a dilute-and-shoot LC-MS-MS assay. J. Anal. Toxicol. 2015, 39, 335-346.

36. Di Rago M, Pantatan S, Hargreaves M, Wong K, Mantinieks D, Kotsos A, Glowacki L, Drummer OH, Gerostamoulos D. High Throughput Detection of 327 Drugs in Blood by LC-MS-MS with Automated Data Processing. J. Anal. Toxicol. 2021, 45, 154-183.

37. Vircks KE, Mulligan CC. Rapid screening of synthetic cathinones as trace residues and in authentic seizures using a portable mass spectrometer equipped with desorption electrospray ionization. Rapid Commun Mass Spectrom. 2012, 26, 2665-2672.

38. Lawton ZE, Traub A, Fatigante WL, Mancias J, O'Leary AE, Hall SE, Wieland JR, Oberacher H, Gizzi MC, Mulligan CC. Analytical Validation of a Portable Mass 
Spectrometer Featuring Interchangeable, Ambient Ionization Sources for High Throughput Forensic Evidence Screening. J Am Soc Mass Spectrom. 2017, 28, 10481059.

39. Pavlovich MJ, Musselman B, Hall AB. Direct analysis in real time-Mass spectrometry (DART-MS) in forensic and security applications. Mass Spectrom Rev. 2018, 37, 171187.

40. Lesiak AD, Musah RA, Cody RB, Domin MA, Dane AJ, Shepard JRE. Direct analysis in real time mass spectrometry (DART-MS) of "bath salt" cathinone drug mixtures. Analyst. 2013, 138, 3424-3432.

41. Grange AH, Sovocool GW. Detection of illicit drugs on surfaces using direct analysis in real time (DART) time-of-flight mass spectrometry. Rapid Commun Mass Spectrom. 2011, 25, 1271-81.

42. Sisco ER, Verkouteren JR, Staymates JL, Lawrence JA. Rapid detection of fentanyl, fentanyl analogues, and opioids for on-site or laboratory based drug seizure screening using thermal desorption DART-MS and ion mobility spectrometry. Forensic Chem., 2017, 4, 108-115.

43. Harper JD, Charipar NA, Mulligan CC, Zhang X, Cooks RG, Ouyang Z. Lowtemperature plasma probe for ambient desorption ionization. Anal Chem. 2008, 80, 9097-104.

44. Jackson AU, Garcia-Reyes JF, Harper JD, Wiley JS, Molina-Díaz A, Ouyang Z, Cooks, R.G. Analysis of drugs of abuse in biofluids by low temperature plasma (LTP) ionization mass spectrometry. Analyst. 2010, 135, 927-933.

45. Liu Y, Lin Z, Zhang S, Yang C, Zhang X. Rapid screening of active ingredients in drugs by mass spectrometry with low-temperature plasma probe. Anal Bioanal Chem. 2009, 395, 591-599.

46. Jett R, Skaggs C, Manicke NE. Drug screening method development for paper spray coupled to a triple quadrupole mass spectrometer. Anal. Methods. 2017, 9, 5037-5043.

47. Kennedy JH, Palaty J, Gill CG, Wiseman JM. Rapid analysis of fentanyls and other novel psychoactive substances in substance use disorder patient urine using paper spray mass spectrometry. Rapid Commun Mass Spectrom. 2018, 32, 1280-1286.

48. Vandergrift GW, Gill CG. Paper spray mass spectrometry: A new drug checking tool for harm reduction in the opioid overdose crisis. J Mass Spectrom. 2019, 54, 729-737. 
49. Vandergrift GW, Hessels AJ, Palaty J, Krogh ET, Gill CG. Paper spray mass spectrometry for the direct, semi-quantitative measurement of fentanyl and norfentanyl in complex matrices. Clin Biochem. 2018, 54, 106-111.

50. McCullough BJ, Patel K, Francis R, Cain P, Douce D, Whyatt K, Bajic S, Lumley N, Hopley C. Atmospheric Solids Analysis Probe Coupled to a Portable Mass Spectrometer for Rapid Identification of Bulk Drug Seizures. J Am Soc Mass Spectrom. 2020, 31, 386-393.

51. Brown HM, McDaniel TJ, Fedick PW, Mulligan CC. The current role of mass spectrometry in forensics and future prospects. Anal Methods. 2020, 12, 3974-3997.

52. www.mzcloud.org

53. Kalant H. The pharmacology and toxicology of "ecstasy" (MDMA) and related drugs. CMAJ. 2001, 165, 917-928.

54. Gahlinger PM. Club drugs: MDMA, gamma-hydroxybutyrate (GHB), Rohypnol, and ketamine. Am Fam Physician. 2004, 69, 2619-2626.

55. Curran HV, Morgan C. Cognitive, dissociative and psychotogenic effects of ketamine in recreational users on the night of drug use and 3 days later. Addiction. 2000, 95, 57590.

56. Benowitz NL. Clinical pharmacology and toxicology of cocaine. Pharmacol Toxicol. 1993, 72, 3-12.

57. Scheinberg MA, Bezerra JB, Almeida FA, Silveira LA. Cutaneous necrotising vasculitis induced by levamisole. Br Med J. 1978, 1, 408.

58. Knip M, Douek IF, Moore WP, Gillmor HA, McLean AE, Bingley PJ, Gale EA; European Nicotinamide Diabetes Intervention Trial Group. Safety of high-dose nicotinamide: a review. Diabetologia. 2000, 43, 1337-1345.

59. Inoue H, Kuwayama K, Iwata YT, Kanamori T, Tsujikawa K, Miyaguchi H. Simple and simultaneous detection of methamphetamine and dimethyl sulfone in crystalline methamphetamine seizures by fast gas chromatography. Forensic Toxicol. 2008, 26, 19-22.

60. Horváth K, Noker PE, Somfai-Relle S, Glávits R, Financsek I, Schauss AG. Toxicity of methylsulfonylmethane in rats. Food Chem Toxicol. 2002, 40, 1459-1462.

61. Karila L, Megarbane B, Cottencin O, Lejoyeux M. Synthetic cathinones: a new public health problem. Curr Neuropharmacol. 2015, 13, 12-20.

62. Borek HA, Holstege CP. Hyperthermia and multiorgan failure after abuse of "bath salts" containing 3,4-methylenedioxypyrovalerone. Ann Emerg Med. 2012, 60, 103-105. 
63. Palamar JJ, Salomone A, Vincenti M, Cleland CM. Detection of "bath salts" and other novel psychoactive substances in hair samples of ecstasy/MDMA/"Molly" users. Drug Alcohol Depend. 2016, 161, 200-205.

64. Department of Health \& Human Services State Government of Victoria. Green 'UPS' pills containing N-ethylpentylone (no MDMA). 2020. Available from: https://www2.health.vic.gov.au/alcohol-and-drugs/drug-alerts/green-ups-containingn-ethylpentylone

65. Department of Health \& Human Services State Government of Victoria. Nethylpentylone in cocaine. 2020. Available from: https://www2.health.vic.gov.au/alcohol-and-drugs/drug-alerts/n-ethylpentylonecocaine

66. Moghimi A, Rahmani S, Zare R, Sadeghzadeh M. Synthesis of 2-(2-Fluorophenyl)-2methylamino-Cyclohexanone as a New Ketamine Derivative. Synth. Commun. 2014, 44, 2021-2028.

67. Wang PF, Neiner A, Lane TR, Zorn KM, Ekins S, Kharasch ED. Halogen Substitution Influences Ketamine Metabolism by Cytochrome P450 2B6: In Vitro and Computational Approaches. Mol Pharm. 2019, 16, 898-906.

68. Taylor JH, Botha CJ, Swan GE, Mülders MS, Grobler MJ. Tiletamine hydrochloride in combination with zolazepam hydrochloride as an anaesthetic agent in sheep. $J$ S Afr Vet Assoc. 1992, 63, 63-65.

69. Chung H, Choi H, Kim E, Jin W, Lee H, Yoo Y. A fatality due to injection of tiletamine and zolazepam. J Anal Toxicol. 2000, 24, 305-308.

70. Morton WA, Stockton GG. Methylphenidate Abuse and Psychiatric Side Effects. Prim Care Companion J Clin Psychiatry. 2000, 2, 159-164.

71. https://www.coronerscourt.vic.gov.au/sites/default/files/202104/COR\%202017\%200216\%20-\%20James.pdf

72. Kueppers VB, Cooke CT. 25I-NBOMe related death in Australia: a case report. Forensic Sci Int. 2015, 249, e15-8.

73. NSW Health. Drug warning: Cocaine and ketamine may contain the dangerous opioids fentanyl and acetylfentanyl. 2020. Available from: https://www.health.nsw.gov.au/aod/public-drug-alerts/Pages/cocaine-or-ketaminecontains-fentanyl.aspx. 
74. NSW Health. Cocaine may contain the dangerous opioids fentanyl and acetylfentanyl. 2020. Available from: https://www.health.nsw.gov.au/aod/public-drugalerts/Pages/cocaine-may-contain-fentanyl-acetylfentanyl.aspx.

75. Lee HM, Wood DM, Hudson S, Archer JR, Dargan PI. Acute toxicity associated with analytically confirmed recreational use of methiopropamine (1-(thiophen-2-yl)-2methylaminopropane). J Med Toxicol. 2014, 10, 299-302.

76. Anne S, Tse R, Cala AD. A Fatal Case of Isolated Methiopropamine (1-(Thiophen-2yl)-2-Methylaminopropane) Toxicity: A Case Report. Am J Forensic Med Pathol. 2015, $36,205-206$. 